\title{
U-REACH: Ultra Reactive Energy Efficient Adaptive Clustering Hierarchical Protocol for Homogeneous and Heterogeneous Wireless Sensor Networks
}

\author{
Komal Sharma \\ M. Tech Student, Department of Computer \\ Science, \\ RCEW, Jaipur, India
}

\author{
Vineet Khanna \\ HOD, Department of Computer Science, \\ RCEW, Jaipur, India
}

\begin{abstract}
A wireless sensor network is a group of sensors heavily distributed in the desired area. There are constraints of energy, distance, mobility etc while dealing in WSN. As we know that randomized algorithms are the best average case providing algorithms so there come the concept of LEACH which is a very well known algorithm in WSN. Wireless sensor networks can be of two kinds: homogeneous sensor network and heterogeneous sensor network. The proposed algorithm provides a better approach for $\mathrm{CH}$ election by increasing network lifetime. It uses 3-level decision tree at different levels of heterogeneity and works in reactive way. Energy balancing is handled by taking average energy of the network as a parameter.
\end{abstract}

\section{Keywords}

Wireless Sensor network, Energy aware, three-level decision tree, heterogeneous, homogeneous, reactive, Clustering, Network lifetime.

\section{INTRODUCTION}

A Wireless Sensor Network (WSN) is a group of a hundreds of sensors with fix energy. Main objective of a wireless sensor network is to identify the defined parameter changes in a given space, receiving data from similar network, after that the data is produced to conclude changes in the area. Sensor nodes have limited power, processing and communication capabilities. Basically sensor nodes have two main functionalities: first to monitor the data changes in the surrounding than sending the sensed changes in data to the sink. Sink is the processing unit with no energy limits like sensor nodes and can handle whole sensed data from a wireless sensor network. Wireless sensor network can be used in many areas such as military, medical care, monitoring temperature, vibration, pressure etc. A sensor basically had four units in its architecture as follows: sensing unit, processing unit, radio unit and the power unit. For power unit sensor nodes have non-rechargeable batteries so the most important issue to handle is to minimize energy consumption. Now the radio unit consumes the most of energy from battery, i.e., the task of sending and receiving messages. Though underlying hardware is improving on its own speed it is better option to improve the quality of routing algorithm working on the same hardware. There could be ways to reduce the communication energy by selection of minimal routes. There are many protocols developed for this purpose but the most significant protocols are hierarchical clustering protocols. By clustering protocols wireless sensor network is divided into group of small clusters that can be residual energy based, randomized, density based, neighbor based, mobility based and many more parameters are there. Custer head $(\mathrm{CH})$ based on the Cluster head $(\mathrm{CH})$ is really important in the performance of a cluster based routing mechanism. LEACH was the earliest work in the hierarchical clustering algorithm. Its randomized approach was the main feature that provides best average case.

In this paper, approach is to improve network lifetime for both homogeneous and heterogeneous wireless sensor network. The paper is organized in 5 sections. In Section II of the paper study of related work is given. In section III proposed algorithm is discussed. In section IV comparative study and simulation results are given. In section $\mathrm{V}$ conclusion and future work details are given.

\section{RELATED WORK}

Many algorithms have been designed to optimize the limited energy of sensors for both homogeneous and heterogeneous WSN. Some of the concerned protocols from both areas are discussed as follows:

\subsection{Low Energy Adaptive Clustering Hierarchy (LEACH)}

It is one of the earliest algorithms in the field of WSN. It used randomization and increased network lifetime to some extent. It is a proactive and hierarchical clustering protocol. The responsibility of cluster head position is rotated between all nodes, due to this, not one node drains its energy early and network remains stable for longer period.

\subsection{I-LEACH}

I-LEACH considers geographical position, energy remaining and no. of neighbors for clustering. This algorithm improves the network lifetime by introducing a new update in the threshold function $\mathrm{T}(\mathrm{n})$ by using average energy of the network, current energy of the node, average no. of neighbors, no. of neighbors of current node, average distance of BS from all nodes, distance of BS from nodes. During data transmission all $\mathrm{CH}$ nodes perform integration of the collected data which reduces energy consumption in data transmission i.e. major reason of battery consumption.

\subsection{Reactive I-LEACH}

This algorithm introduces the concept of 3-level decision tree for cluster head selection in which at first level nodes with higher energy than the average energy of network are passed to the next level where node with minimum distance to each other are passed to next level and at last level nodes are elected same as in LEACH. During cluster formation BS is placed at the center of the quadrant which has maximum no. of nodes. During data transmission it works in reactive way. 


\subsection{Stable Election Protocol (SEP)}

This was the first protocol which introduced energy heterogeneity in WSN. In total no. of nodes $n, m$ fraction of nodes have more energy by factor $\alpha$. In the place of optimal election probability election probabilities with weights are used for normal and advanced nodes.

\subsection{Threshold Sensitive Energy Efficient sensor network protocol (TEEN)}

It introduced reactive routing approach. It uses the concept of hard threshold and soft threshold. In this, first time nodes transmit data when value is more than hard threshold and second time it sends data when the difference between the present and past values is more than the soft threshold.

\subsection{Threshold Sensitive Stable Protocol (TSEP)}

TSEP proposed three level of heterogeneity and protocol works in reactive way. Three kinds of nodes: normal, intermediate and advanced nodes on the basis of their energy level are taken and weighted election probability is given.

\subsection{Ultra Stable Threshold Sensitive Election Protocol for Mobile WSN (USEP)}

USEP proposes five levels of heterogeneity and similar to other heterogeneous protocols, cluster head election is done on the basis of threshold function T (n) From LEACH.

\section{PROPOSED ALGORITHM}

As per the above discussed in Section II very less work is done in the direction of common solution for homogeneous and heterogeneous WSN. This algorithm deals with energy heterogeneity of sensor nodes. Nodes can be of same energy or there can be nodes with multiple energy levels. Here is proposed a more efficient algorithm, new approach to handle homogeneity and heterogeneity in the same environment. This algorithm is applicable for WSN with following specification:

- Nodes can have same energy (homogeneous) or multiple energy (heterogeneous), but with not rechargeable batteries. Nodes die with the end of their batteries.

- Energy heterogeneity cannot exceed 5 level of heterogeneity i.e. there can be 5 types of nodes by their level of energy.

- Sensor nodes are not mobile. They remain in the same place during their lifetime.

- Sensor unit is independent and vary to a complete range of available solutions

- A mini-BS (Base Station) router is placed in the wireless sensor area after sensor deployment which is responsible for all communication between base station and wireless sensor area.

Just like other hierarchical clustering protocols there are three phases in every routing algorithm. In addition to that, this algorithm is also placing a mini-BS router in the sensor deployed area with one more phase named network initialization [4]. Since this algorithm is working for both homogeneous and heterogeneous WSN in terms of energy it will describe all these phases in both references.

\subsection{Network Initialization Phase}

In network initialization phase all nodes are randomly placed and after sensor deployment nodes send their position detail to the main BS. Main Base Station divides the whole area into four quadrants and calculates that which quadrant is densest, after this Main BS places the mini-BS at the center of the densest quadrant [4]. Now Main BS broadcast the coordinates of mini BS router and all further communication of nodes is handled by mini BS router which has no battery limitations and can work for longer period than the network lifetime. Mini BS router plays a role of mediator which conveys messages.

\subsection{Cluster Heads Selection Phase}

\subsubsection{For Heterogeneous setup}

Following factors are taken to define nodes : (1)Energy coefficient $(\alpha)$ It is minimum amount of energy by which nodes will differ in their energy levels; (2) Advanced nodes percentage (m) ; (3) Intermediate nodes percentage (b). In case of heterogeneity, value of $\alpha$ will vary and values of $m$ and $b$ will also be considered. In heterogeneity, nodes with different energy will have different weighted election probability as is done in [5][6][7]. For 5 levels of heterogeneity, nodes are
1) Normal (nrm)
2) Subnormal (subnrm)
3) Intermediate (int)
4) Advanced (adv)
5) Super (sup)

To define specific details, the energy of all nodes is as follows

$\mathrm{E}(\mathrm{nrm})=E_{o} ; \mathrm{E}(\mathrm{Sub})=E_{o}(1+\mu / 2) ; \mathrm{E}(\mathrm{int})=E_{o}(1+\mu) ; \mathrm{E}(\mathrm{adv})$ $=E_{o}(1+\alpha) ; \mathrm{E}($ super $)=E_{o}(1+2 \alpha)$; Here $\mu=\alpha / 2$;

(1)

The ratio of different nodes will be calculated by the values of $m$ and $b$; for instance $n=$ Total no of nodes; $m=$ fraction of advanced nodes; $b=$ fraction of intermediate nodes. On the basis of probability distribution in previous heterogeneous algorithms like [5][6][7], and on the basis of energy election probability of different nodes will be as follows:

$$
\begin{aligned}
& P_{\text {nrm }}=\left\{\frac{P_{\text {opt }}}{[1+m \alpha+b \mu]}\right\} ; P_{\text {subnrm }}=\left\{\frac{P_{\text {opt }}(1+\mu / 2)}{[1+m \alpha+b \mu]}\right\} ; \\
& P_{\text {int }}=\left\{\frac{P_{\text {opt }}(1+\mu)}{[1+m \alpha+b \mu]}\right\} ; P_{a d v}=\left\{\frac{P_{\text {opt }}(1+\alpha)}{[1+m \alpha+b \mu]}\right\} ; \\
& P_{\text {sup }}=\left\{\frac{P_{o p t}(1+2 \alpha)}{[1+m \alpha+b \mu]}\right\} ;
\end{aligned}
$$

\subsubsection{For Homogeneous setup}

The value of $\alpha$ will be 1 and $m=0, b=0$.

Election probability will be $p_{\text {opt }}$ i.e. same as in [1][2][3]. After the assignment of election probability both homogeneous network nodes and heterogeneous network nodes are passed to the 3-level decision tree and resultant nodes are considered cluster heads $(\mathrm{CH})$ for that particular round. All nodes go through this decision tree in every round.

At level I average energy of the network is calculated. Nodes which have higher or equal energy than the average energy are passed to the level 2 . 
As shown in Figure 1 at level-2 density equalizer (d) [4][8]is calculated as follows;

$$
d=\frac{1}{\sqrt{\pi}} \frac{2 M}{\sqrt{\frac{\sqrt{N} \sqrt{\epsilon_{f s}}}{\sqrt{\pi} \sqrt{\epsilon_{m p}} d_{\text {miniBS }}}}}
$$

Where $\mathrm{M}=$ area of the sensor field, $\mathrm{N}=$ total no of nodes in the field; $d_{\text {miniBS }}=$ average distance of all nodes from the mini BS router.

Now the nodes of level 2 which have $d$ minimum distance from mini BS router and minimum d distance from other nodes of level 2 are passed to level 3 of the decision tree

At level-3 the basic LEACH functionality will be applied. Each node which has passed level 2 is given a random no between $\{0,1\}$. Now threshold function $T(n)$ will be calculated.

At this level $T(n)$ for homogeneous WSN will be calculated by using $p_{\text {opt }}$ as follows:

$T(n)=\frac{p_{\text {opt }}}{\left(1-p_{\text {opt }}\left(\operatorname{rmod}\left(\frac{1}{p_{\text {opt }}}\right)\right)\right)}$

And in case of heterogeneous WSN for normal, subnormal, intermediate, advanced and super to calculate $T(n)$ :

$P_{n r m}, P_{\text {subnrm }}, P_{\text {int }}, P_{\text {adv }}, P_{\text {sup }}$ will be used respectively.

Now the random values assigned to the nodes will be compared to the respective $T(n)$, if node has less value then it becomes the cluster head.

\subsection{Cluster Formation Phase}

We have already placed our mini BS router at the center of densest area by which energy of many nodes is saved in terms of communication [4]. Now nodes which are not cluster heads select their $\mathrm{CH}$ by considering "d" radius. Nodes which are in d radius of mini BS router communicate directly to mini BS. Other clusters are formed respectively as shown in figure.

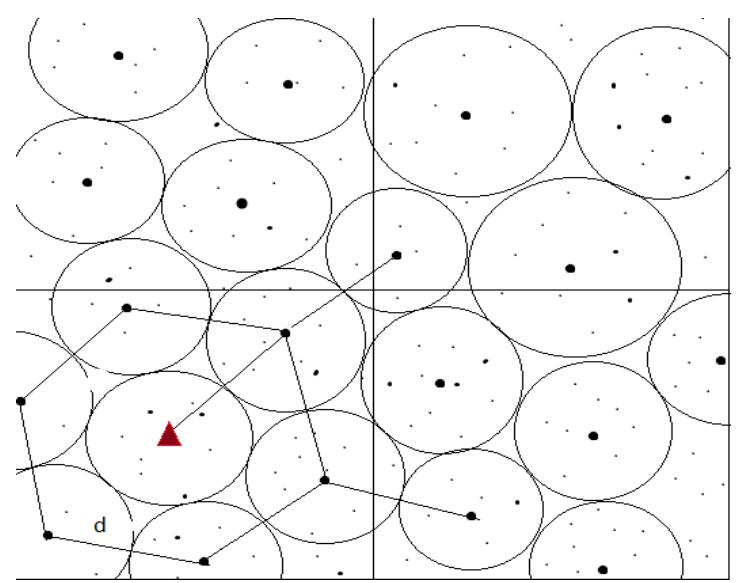

Fig. 1.Density equalized Cluster formation in U-REACH

\subsection{Data transmission}

$\mathrm{U}-\mathrm{REACH}$ is a reactive protocol. Nodes in the sensor area are continuously sensing i.e. their sensing unit keeps working while other units remain in sleep state [5]. When sensor unit identify some significant changes in the sensor area it sends data to the respective $\mathrm{CH}$. It saves a lot of data transmission energy.
The complete algorithm is given below.

\section{U-REACH}

Step I: Sensor area is divided into four equal parts and mini BS router is placed in the densest area

Step II: For round 1: R repeat steps II to VI

Step III: If nodes are of multiple energy levels then they are given different election probabilities as in equation 2 .

Step IV: Nodes with energy more than average energy are listed in level 1 list.

Step V: Nodes from level1 list which have distance (based on density equalizer in eq. (3)) from mini BS and other nodes from level-1 list are listed in level-2 list.

Step VI: T(n) is calculated for each node from level 2 list and nodes which have this value less than the value of T(n) calculated from eq. (4) is selected as cluster head.

\section{SIMULATION}

\subsection{Used Energy Model}

Energy model is same as in [1]. Equation for it is as follows:

$$
\begin{gathered}
E_{T X}(k, d)= \begin{cases}k E_{\text {elec }}+k \varepsilon_{f s} d^{2}, & d<d_{0} \\
k E_{\text {elec }}+k \varepsilon_{m p} d^{4}, & d \geq d_{0}\end{cases} \\
d_{0}=\sqrt{\left(\frac{\varepsilon_{f s}}{\varepsilon_{m p}}\right)} \quad \text { and } \quad E_{R X}(k)=k E_{\text {elec }}
\end{gathered}
$$

Here, $\mathrm{k}$ is the size of the data packet; $\mathrm{d}$ is distance covered by data packet; $E_{T X}$ is data transmission energy; $E_{R X}$ is data receiving energy; $E_{\text {elec }}$ is the energy consumption of electronic circuits.

\subsection{Performance measurement}

Three performance measurements are considered as follows [5]:

1. Stability period: duration from the starting of network to the first node dead round.

2. Instability Period: duration from the first node dead round to the last node dead round.

3. Network Lifetime: duration from starting of network to the last node dead round.

\subsection{Network Parameters and Simulation}

MATLAB is used for network simulation. Table 1 describes the network parameters for the simulation. In simulation the value of energy coefficient $\alpha$, in homogeneous WSN is taken 1 and in Heterogeneous WSN is taken $1 \& 4$. The values of $\mathrm{m} \& \mathrm{~b}$ will be considered 0 in homogeneous settings and in heterogeneous settings $\mathrm{m}=0.2 \& 0.3$ and $\mathrm{b}=0.4$ is taken.

Figure 2 shows the network in active state. Figure 3 shows comparison $\mathrm{b} / \mathrm{w}$ different homogeneous algorithms in respect to the first node dead round and last node dead round. Figure 4 shows comparison between different heterogeneous algorithms in respect to the first node dead round and last node dead round for $\alpha, \mathrm{m} \& \mathrm{~b}$ respectively $(1,0.2,0.3)$. 
Figure 5 shows alive nodes v/s iterations for homogeneous setup in U-REACH. Figure $6 \& 7$ shows alive nodes v/s iterations for heterogeneous setup for two sets SET1 (1, 0.2, $0.4)$ and SET2 $(4,0.3,0.4)$ respectively.

Figure 8 shows throughput v/s iterations for homogeneous setup. Figure 9 and 10 shows throughput v/s iterations for heterogeneous setup for two sets SET1 $(1,0.2,0.4)$ and SET2 $(4,0.3,0.4)$ respectively.

\subsection{Results and analysis}

In this algorithm density equalization with decision tree generation has improved the performance of the network. For performance evaluation of proposed technique we have compared it with two sets of algorithms one is set of homogeneous algorithms like LEACH, I-LEACH and reactive I-LEACH, second is the set of heterogeneous algorithms like SEP, TSEP and USEP. Figure 3 and 4 shows the first node and last node dead round comparison for homogeneous and heterogeneous set 1 setup respectively. UREACH increases network lifetime in terms of first node dead round. It improves performance of LEACH by $198.19 \%$, I-LEACH by $88.06 \%$, reactive I-LEACH by $22.52 \%$, SEP by $305 \%$, TSEP by $23.54 \%$, USEP by $5 \%$. The significance of this algorithm is that it provides a common solution for both homogeneous and heterogeneous networks with improved performance compare to the other as above.

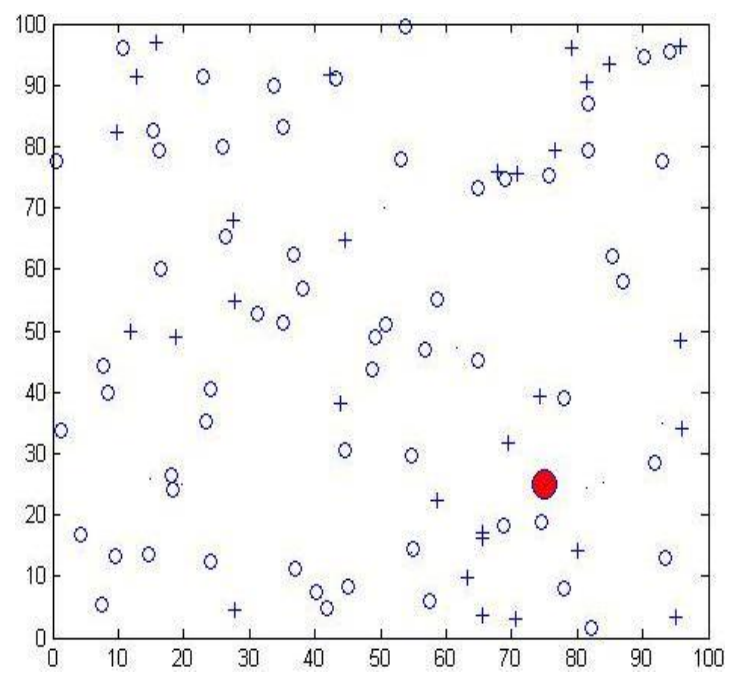

Figure 2.Network in active state

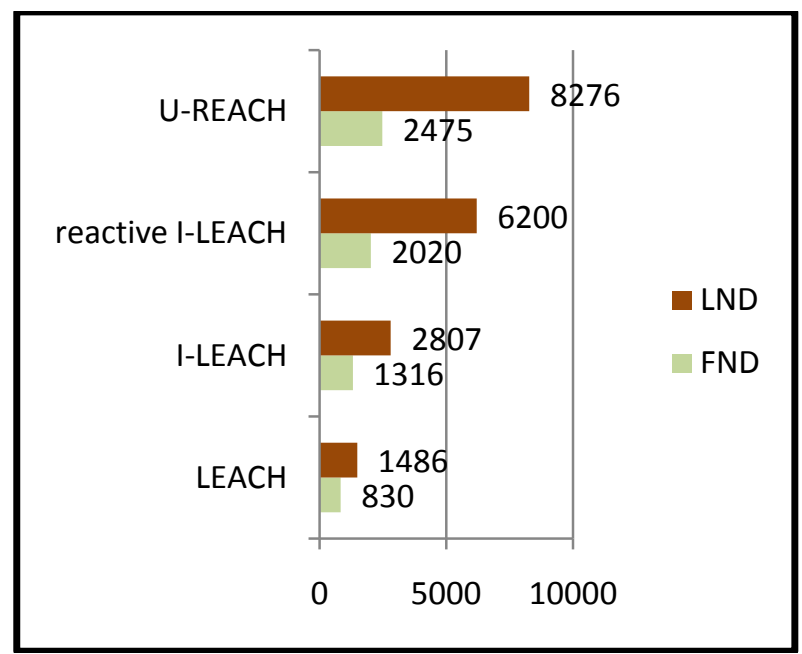

Figure. 3.WSN First and last node dead round for

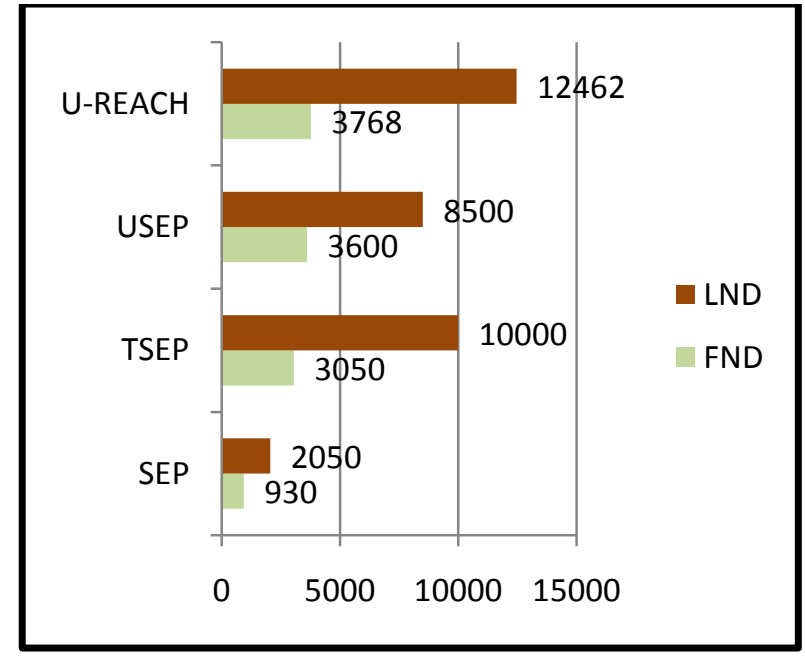

Figure 4.WSN FND and LND for Heterogeneous setup

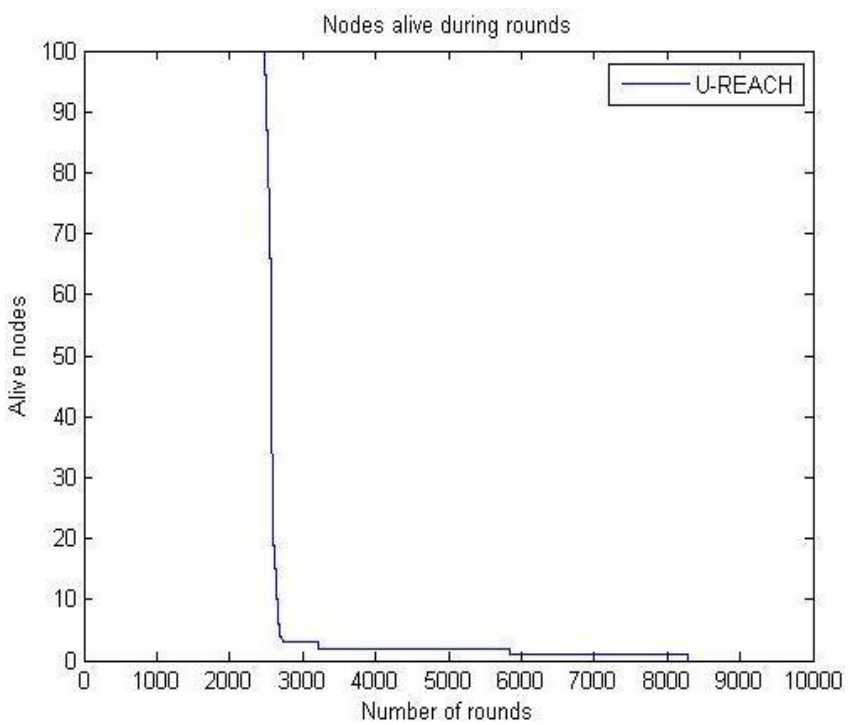

Figure 5.Alive nodes per round in homogeneous setup

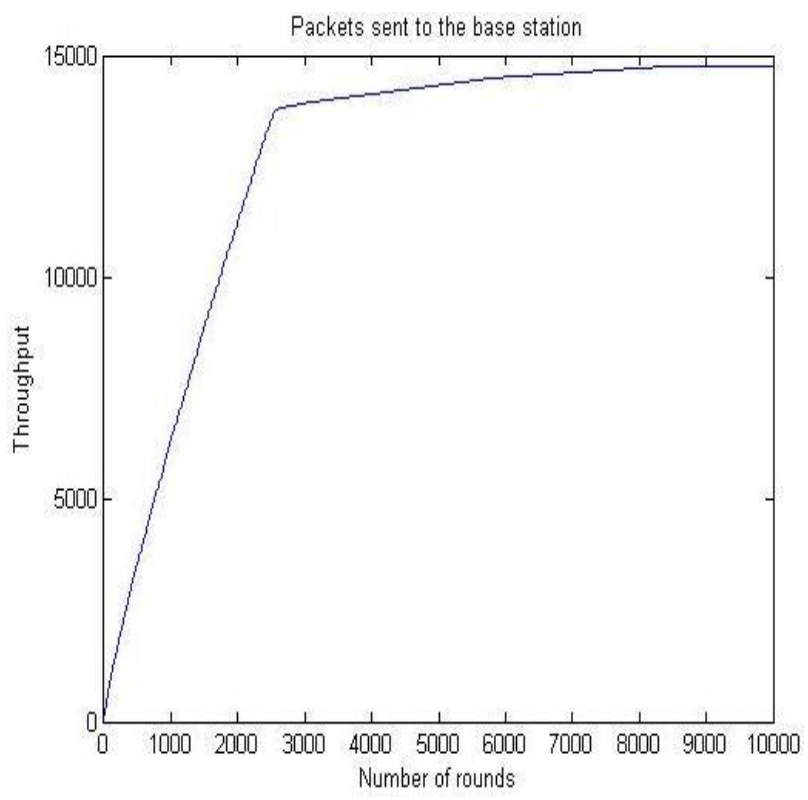

Figure 6.Throughput for homogeneous setup 


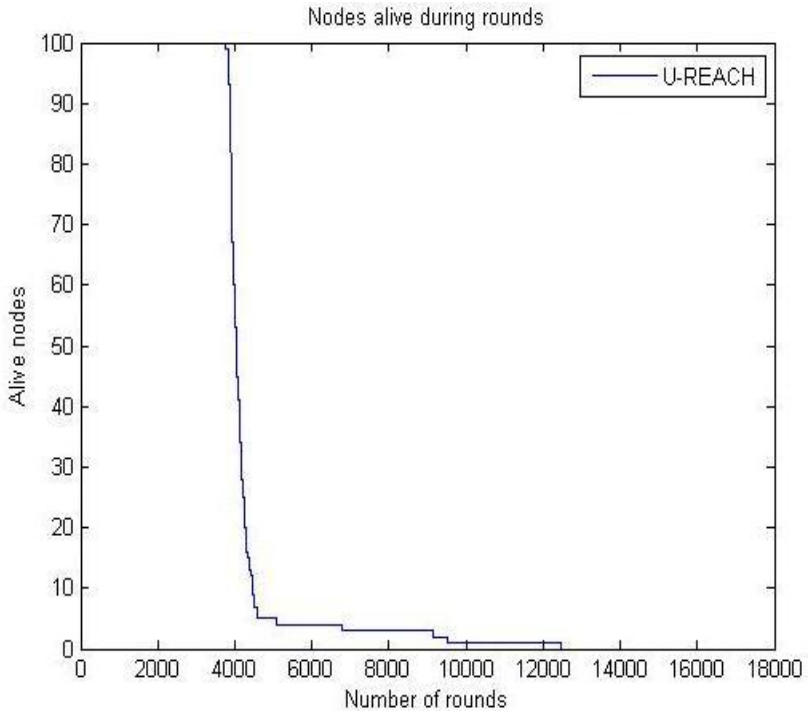

Figure 7.Alive nodes per round in heterogeneous set1

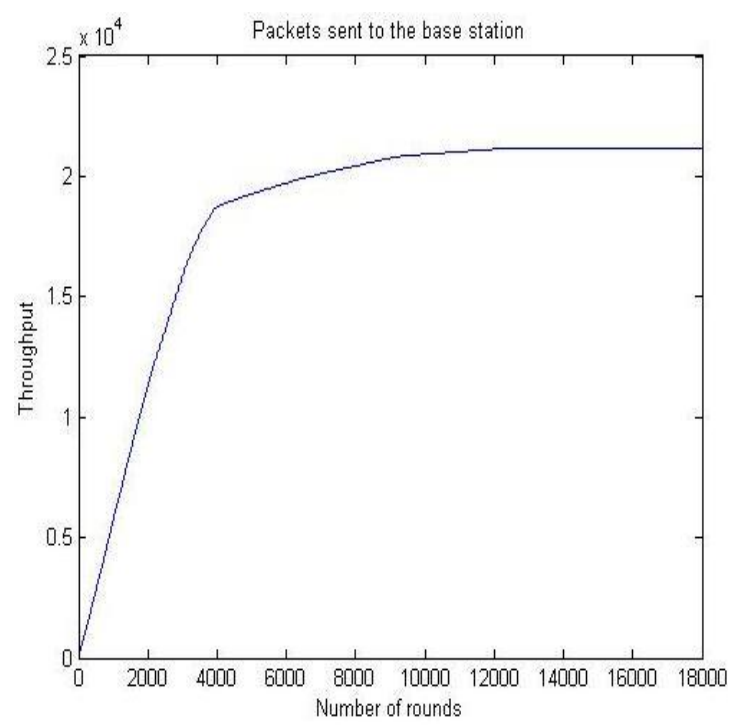

Figure 8.Throughput for heterogeneous set 1

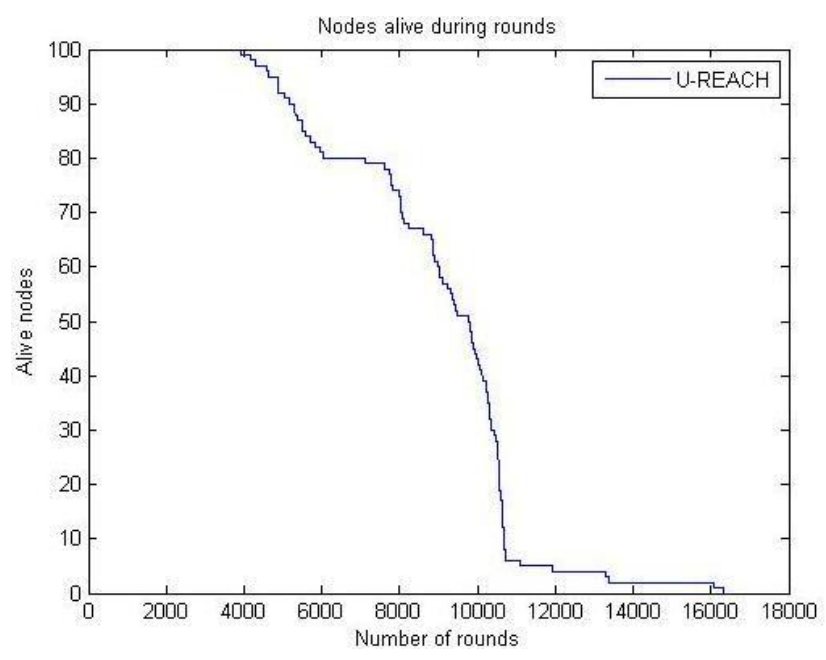

Figure 9.Alive nodes per round in heterogeneous set 2

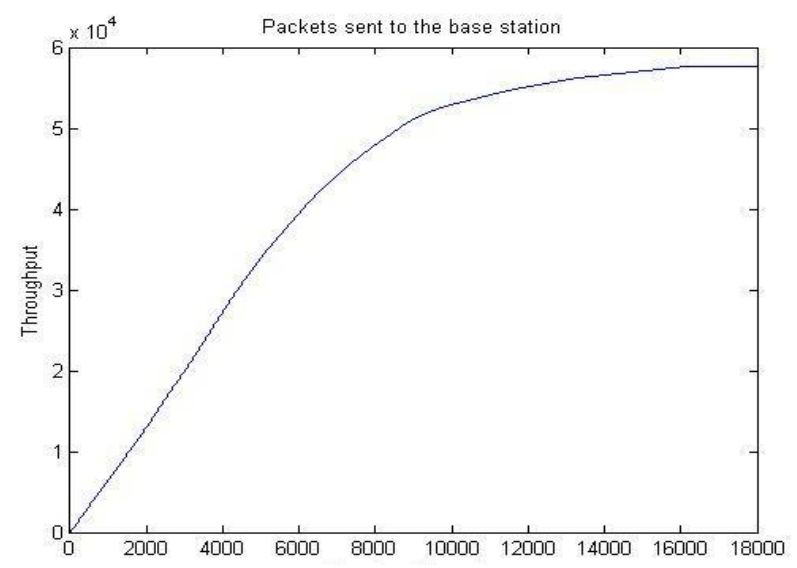

Figure 10.Throughput in heterogeneous set 2

Table 1. Simulation parameters

\begin{tabular}{|c|c|}
\hline Parameter name & Values \\
\hline Network Size & $100 * 100$ \\
\hline Number of nodes(N) & 100 \\
\hline Probability $\left(P_{\text {opt }}\right)$ & 0.1 \\
\hline $\mathrm{E}$ & $0.5 \mathrm{~J}$ \\
\hline Free Space $\left(E_{f s}\right)$ & $10 \mathrm{pj} / \mathrm{bit} / \mathrm{m}^{\wedge} 2$ \\
\hline Multipath fading $\left(E_{m p}\right)$ & $0.0013 \mathrm{pj} / \mathrm{bit} / \mathrm{m}^{\wedge} 2$ \\
\hline$E_{\text {elect }}$ & $50 \mathrm{~nJ} / \mathrm{bit}$ \\
\hline The energy of & $5 \mathrm{NJ} / \mathrm{bit} / \mathrm{signal}$ \\
\hline aggregation $\left(E_{D A}\right)$ & $4000 \mathrm{bits}, 6000 \mathrm{bits}$ \\
\hline Data packet size & 1,4 \\
\hline$\alpha$ & $0,0.2,0.3$ \\
\hline $\mathrm{m}$ & $0,0.4$ \\
\hline $\mathrm{b}$ & \\
\hline
\end{tabular}

\section{CONCLUSION \& FUTURE WORK}

Results show that proposed changes can help in providing a more dynamic and more efficient solution for wireless sensor networks. It supports multi level heterogeneity up to 5 levels. So the network with 5 different kinds of nodes (i.e. in terms of energy) can use this protocol and at the same time a completely homogeneous sensor network can use this. Thus single protocol can be used to handle multiple networks by a centralized base station. Energy balancing, load balancing, 5levels of heterogeneity with support to homogeneity and heterogeneity in sensor node energy all are summed at one place. BS positioning is done on the basis of density. Proposed algorithm is not dynamic; in future more dynamic solution will be developed that will support 0 to 5 level of heterogeneity. The scope of development is wide as the placement of mini BS router, the three level decision tree can be extended to higher levels, more parameters can be considered, density equalization can be more optimized, proposed algorithm can be improved for more dynamic and robust solutions. This approach is implemented on static nodes with fixed energy values. Further development can be done for mobile nodes with dynamic energy values. There is wide array of development possible. BS positioning will be more optimized in future. 


\section{REFERENCES}

[1] W.R. Heinzelman, A. Chandrakasan, and H. Balakrishnan, "Energy efficient communication protocol for wireless micro sensor networks" IEEE Proceedings of the 33rd Hawaii International Conference on System Sciences, pp. 3005-3014, Jan 2000 .

[2] Z. Beiranvand, A. Patooghy and M. Fazeli, "I-LEACH: An efficient routing Algorithm to Improve Performance $\&$ to Reduce Energy Consumption in Wireless Sensor Networks", IEEE 5th International Conference on Information and Knowledge Technology, pp. 13-18, May2013.

[3] M. Shankar, "Performance Evaluation of LEACH Protocol in Wireless Network" International Journal of Scientific \& Engineering Research, Volume 3, Issue 1, January-2012.

[4] V. Kehar, "Evaluating the performance of Reactive ILEACH", 2014 International Conference on Advances in Computing, Communication and Informatics, IEEE 2014

[5] G. Smaragdakis, "SEP: A Stable Election Protocol For clustered heterogeneous wireless sensor networks" Boston.

[6] A. Kashaf, N. Javaid, Z. A. Khan and I. A. Khan, "Threshold sensitive Stable Election Protocol for WSNs," in IEEE, pp. 164-168, Dec. 2012.

[7] S. Verma, "USEP: Ultra Stable Threshold Sensitive Election Protocol for Mobile WSN" International Journal of Computer Applications, Volume 100 - no. 3 , August 2014.

[8] Z. Xu, "An Density based Energy-efficient routing Algorithm in Wireless sensor networks using game theory", International journal of future Generation Communication and Networking, Vol. 5, No. 4, December, 2012. 